\title{
Ubiquitin levels: the next target against gynecological cancers?
}

\author{
Diane L. Haakonsen and Michael Rape \\ Department of Molecular and Cell Biology and Howard Hughes Medical Institute, University of California at Berkeley, Berkeley, California, USA.
}

\begin{abstract}
Ubiquitylation is a tightly regulated process that is essential for appropriate cell survival and function, and the ubiquitin pathway has shown promise as a therapeutic target for several forms of cancer. In this issue of the $\mathrm{JCI}$, Kedves and colleagues report the identification of a subset of gynecological cancers with repressed expression of the polyubiquitin gene UBB, which renders these cancer cells sensitive to further decreases in ubiquitin production by inhibition of the polyubiquitin gene UBC. Moreover, inducible depletion of $U B C$ in mice harboring tumors with low $U B B$ levels dramatically decreased tumor burden and prolonged survival. Together, the results of this study indicate that there is a synthetic lethal relationship between UBB and $U B C$ that has potential to be exploited as a therapeutic strategy to fight these devastating cancers.
\end{abstract}

\section{Ubiquitylation: a tightly regulated process}

All eukaryotic cells rely on ubiquitylation - the attachment of ubiquitin to a target protein - to control a vast array of processes, including protein degradation, DNA repair, transcription, protein trafficking, cell-cycle regulation, and signal transduction. As many of these processes are essential for the survival of cancer cells, components of the ubiquitin pathway have emerged as attractive therapeutic targets for cancer treatment. Ubiquitin is a highly conserved, 76-amino acid protein that is estimated to represent up to $5 \%$ of the total cellular protein content (1). Ubiquitin can be transferred to its cellular targets either as a single molecule, referred to as monoubiquitylation, or as polymeric ubiquitin chains. Ubiquitin chains can differ in topology and function. For example, homotypic ubiquitin chains connected through Lys11 (K11) or Lys48 (K48) of one ubiquitin and the carboxy terminus of the next ubiquitin molecule trigger degradation of substrate proteins by the $26 \mathrm{~S}$ pro- teasome, whereas K63- or Met1-linked ubiquitin chains act as a molecular glue that allows reversible assembly of large signaling complexes (2). Multiple ubiquitin linkages can also be combined in a single conjugate to generate mixed or branched chains, and K11/K48-branched chains were recently found to work as priority access signals for proteasomal degradation of aggregation-prone proteins (3). While proteolytic ubiquitin chains are essential signals for cell cycle and protein quality control, nonproteolytic ubiquitin marks control protein sorting as well as inflammatory signaling pathways. This cellular signaling language requires precise regulation of the necessary enzymes, including the approximately 600 human E3 ubiquitin ligases that recruit specific substrates, as well as high concentrations of the modifier itself, ubiquitin.

The high micromolar concentration of ubiquitin in cells is achieved by expression from four genes. RPS $27 A$ and UBA52 encode ubiquitin as in-frame fusions to a small and large ribosomal protein, respec-

Related Article: p. 4554

Conflict of interest: M. Rape is cofounder and consultant to Nurix, a company working in the ubiquitin space.

Reference information: J Clin Invest. 2017;127(12):4228-4230. https://doi.org/10.1172/JCI98262.

tively, whereas $U B B$ and $U B C$ encode fusions of three and nine ubiquitin molecules, respectively (4). The monomeric ubiquitin modifier is generated from these precursors by the activity of deubiquitylases (DUBs), such as USP5, which selectively cleave ubiquitin molecules off of their fusion partners (5). DUBs also recycle ubiquitin after its signaling function has been fulfilled, and depletion of proteasome- or lysosome-associated DUBs leads to degradation of ubiquitin along with its targets, resulting in depletion of cellular ubiquitin levels $(6,7)$. All four ubiquitin genes contribute to basal ubiquitin levels; however, $U B B$ and $U B C$ are upregulated in response to cellular stresses, such as heat shock or oxidative stress (8). Increased ubiquitin expression from these loci is essential for metazoan development, as UBC deletion in mice is embryonic lethal due to defective hepatocyte differentiation and $U B B$ deletion causes infertility and adult-onset neurodegeneration $(9,10)$. Although ubiquitin is essential in all eukaryotic cells, the ubiquitin levels that guarantee cellular homeostasis and the contributions of $U B B$ and $U B C$ may be different in distinct tissues. Whether the cellular machinery that establishes proper ubiquitin levels could be targeted for therapeutic benefit has been unclear.

\section{Stable UBB suppression in aggressive gynecological cancers}

In this issue, Kedves et al. analyzed expression data across 27 tumor types and found that expression of $U B B$ was specifically decreased in high-grade serous ovarian cancers (HGSOCs), while other ubiquitin-encoding loci were not affected (11). Moreover, $U B B$ expression correlated with the methylation status of the gene, implicating methylation as a $U B B$-silencing mechanism. Using the $U B C / U B B$ mRNA ratio as a measure of $U B B$ silencing, Kedves and colleagues showed that three gynecological cancers (uterine carcinosarcomas, 


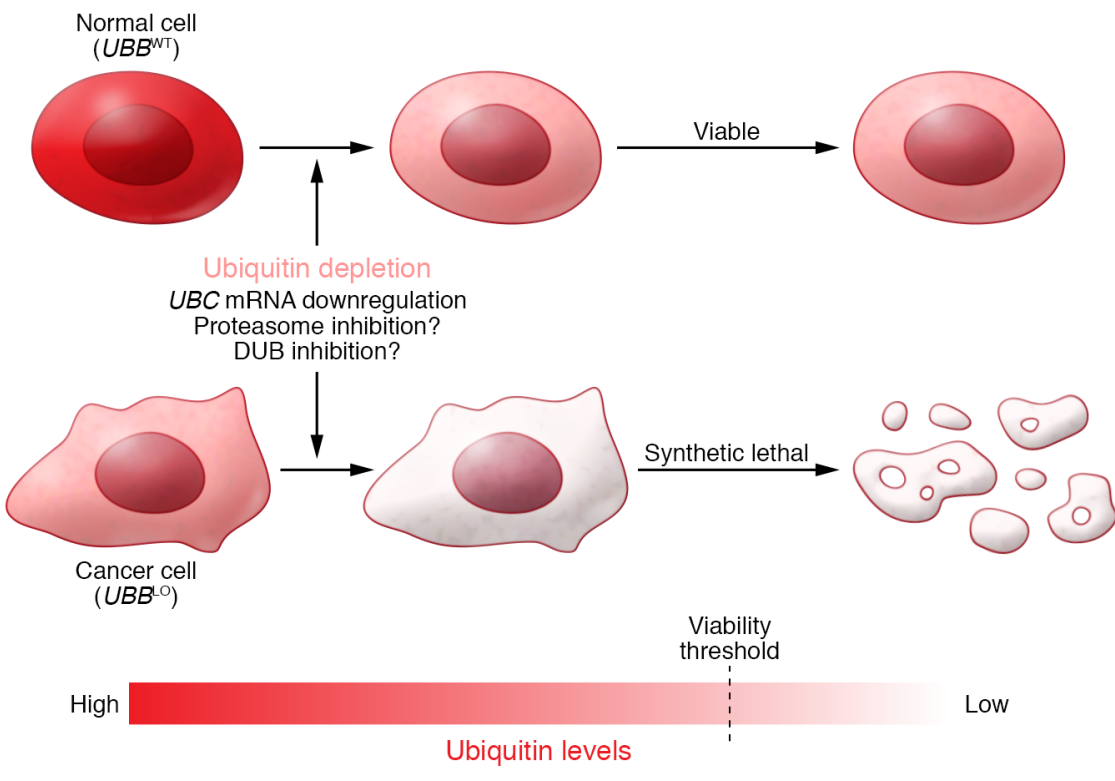

uterine corpus endometrial carcinomas, and HGSOC), all derived from Müllerian epithelium, display a high prevalence of $U B B$ silencing. Moreover, compared with those with average $U B B$ expression, HGSOC samples with low $U B B$ expression associated with poorer patient survival outcomes, suggesting $U B B$ silencing may play a role in progression of this cancer.

Extraction of data from the Cancer Cell Line Encyclopedia yielded similar results, as the majority of cell lines with low $U B B$ expression were derived from gynecological cancers. Based on these observations, Kedves et al. wondered whether low expression of $U B B$ would sensitize cells to a further decrease in ubiquitin levels, and thereby provide a potential therapeutic strategy against these cancers. Indeed, depletion of the polyubiquitin $U B C$ gene affected viability of cell lines with low $U B B$ expression but not those in which $U B B$ was expressed at normal levels (Figure 1). This observation suggested that cancer cells with a repressed $U B B$ locus could be selectively eliminated by downregulation of the UBC gene. Kedves and colleagues tested this notion by implanting gynecological cancer cells with low levels of $U B B$ and an inducible system to deplete $U B C$ into immunodeficient mice. Depletion of $U B C$ resulted in marked tumor regression and a long-term survival benefit, as compared with control animals. Strikingly, all resistant tumors were the result of a lack of $U B C$ knockdown and not due to a release of $U B B$ silencing, indicating that downregulation of $U B B$ expression is a stable repressive mechanism. Together, these findings provide a solid foundation for targeting $U B C$ as a therapeutic intervention for these high-grade uterine cancers.

It is not clear why $U B B$ silencing appears to predominantly affect gynecological cancers. Cancer cells evolve rapidly, often leading to aneuploidy or a high mutational load, both of which increase the need for ubiquitin-dependent proteasomal or autophagosomal degradation $(12,13)$. Similarly, ubiquitylation controls many important cell fate specification events during early development $(14,15)$. These observations suggest that different ubiquitin levels may be critical in distinct cellular contexts, providing a potential explanation for the striking observation that gynecological cancers in particular may profit from stable repression of the $U B B$ gene. Although Kedves et al. did not directly show that low $U B B$ expression alters cellular ubiquitin levels, lack of $U B B$ expression could alter the dynamics of ubiquitin production, and most likely, leads to lower ubiquitin abundance. Protein quality control, cell cycle progression, and DNA damage all depend on extensive ubiquitylation, often involving branched ubiquitin chains with many ubiquitin molecules; therefore, a partial inhibition of such regulatory networks could result in an evolutionary advantage for certain cancer cells. Further studies are needed to identify the mechanisms that
Figure 1. Further depletion of ubiquitin levels in cancer cells with low levels of UBB expression results in synthetic lethality. Cancer cells with repressed expression of the UBB polyubiquitin gene $\left(U B B^{L O}\right)$ likely have lower cellular ubiquitin levels and, therefore, are sensitive to further depletion of ubiquitin by inhibition of the polyubiquitin gene UBC. In contrast, cells with normal ubiquitin levels (UBB ${ }^{\mathrm{WT}}$ ) remain viable after depletion of $U B C$. Alternatively, proteasome and DUB inhibitors could constitute effective treatment options for cancers with reduced ubiquitin, as these inhibitors also lower ubiquitin levels. favor lower ubiquitin levels, as those pathways could provide additional therapeutic targets against these cancers.

\section{Conclusions}

Ubiquitin has recently taken center stage for cancer drug discovery. This spotlight has been especially driven by the discovery that thalidomide and its derivatives, which are effective against multiple myeloma, alter the substrates targeted by the cereblon-containing E3 ubiquitin ligase (16). To date, most approaches have focused specifically on targeting the enzymes involved in the ubiquitylation reaction, such as tumor-related E3 ligases like MDM2. However, the work by Kedves et al. indicates that strategies to target ubiquitin-encoding genes have potential for therapeutic benefit against some cancers. Specifically, this study points to a synthetic lethal relationship between $U B B$ and $U B C$ that can serve as an entry to treat a subset of aggressive gynecological cancers (Figure 1). Strategies to target $U B C$ at the mRNA level will need to be developed and may include the use of siRNA therapeutics or antisense oligonucleotides; however, the systemic effects, such as toxicity, of $U B C$ silencing remain to be assessed. Alternatively, inhibition of either the proteasome or DUBs have been shown to lower the ubiquitin levels in cells and might therefore be effective in gynecological cancers. Learning more about the connectivity of ubiquitin signaling networks in gynecological cancer cells could therefore be a fruitful strategy 
for discovering new approaches that benefit patients of these often aggressive and difficult-to-treat pathologies.

Address correspondence to: Diane L. Haakonsen, Department of Molecular and Cell Biology, 330F Li Ka Shing Center, University of California at Berkeley, Berkeley, California 94720, USA. Phone: 510.642.0468; Email: haakonsen@berkeley.edu.

1. Park CW, Ryu KY. Cellular ubiquitin pool dynamics and homeostasis. BMB Rep. 2014;47(9):475-482.

2. Komander D, Rape M. The ubiquitin code. Annu Rev Biochem. 2012;81:203-229.

3. Yau RG, et al. Assembly and function of heterotypic ubiquitin chains in cell-cycle and protein quality control. Cell. 2017;171(4):918-933.

4. Finley D, Bartel B, Varshavsky A. The tails of ubiquitin precursors are ribosomal proteins whose fusion to ubiquitin facilitates ribosome biogenesis. Nature. 1989;338(6214):394-401.

5. Reyes-Turcu FE, Ventii KH, Wilkinson KD. Regulation and cellular roles of ubiquitin-specific deubiquitinating enzymes. Annu Rev Biochem. 2009;78:363-397.

6. Song L, Rape M. Reverse the curse - the role of deubiquitination in cell cycle control. Curr Opin Cell Biol. 2008;20(2):156-163.

7. Leggett DS, et al. Multiple associated proteins regulate proteasome structure and function. $\mathrm{Mol}$ Cell. 2002;10(3):495-507.

8. Finley D, Ozkaynak E, Varshavsky A. The yeast polyubiquitin gene is essential for resistance to high temperatures, starvation, and other stresses. Cell. 1987;48(6):1035-1046.

9. Ryu KY, Garza JC, Lu XY, Barsh GS, Kopito RR. Hypothalamic neurodegeneration and adult-onset obesity in mice lacking the Ubb polyubiquitin gene. Proc Natl Acad Sci U S A 2008;105(10):4016-4021.

10. Ryu KY, et al. The mouse polyubiquitin gene $\mathrm{UbC}$ is essential for fetal liver development, cell-cycle progression and stress tolerance. EMBO J. 2007;26(11):2693-2706

11. Kedves AT, et al. Recurrent ubiquitin B silencing in gynecological cancers establishes dependence on ubiquitin C. J Clin Invest. 2017; 127(12):4554-4567

12. Dodgson SE, Santaguida S, Kim S, Sheltzer J, Amon A. The pleiotropic deubiquitinase Ubp3 confers aneuploidy tolerance. Genes Dev. 2016;30(20):2259-2271.

13. Oromendia AB, Dodgson SE, Amon A. Aneuploidy causes proteotoxic stress in yeast. Genes Dev. 2012;26(24):2696-2708.

14. Rape M. Ubiquitylation at the crossroads of development and disease [published online ahead of print September 20, 2017]. Nat Rev Mol Cell Biol.https://doi.org/10.1038/nrm.2017.83.

15. Werner A, et al. Cell-fate determination by ubiquitin-dependent regulation of translation. Nature. 2015;525(7570):523-527.

16. Huang X, Dixit VM. Drugging the undruggables: exploring the ubiquitin system for drug development. Cell Res. 2016;26(4):484-498. 